\title{
Abdominal Wall Reconstruction Utilizing the Combination of Absorbable and Permanent Mesh in a Retromuscular Position: A Multicenter Prospective Study
}

\author{
Miguel Ángel García-Ureña, \\ Phone: + 34619051622 \\ Email: magurena@gmail.com
}

Javier López-Monclús, 2

Diego Cuccurullo, 4

Luis Alberto Blázquez Hernando,

Providencia García-Pastor, 3

Stefano Reggio, 4

Elena Jiménez Cubedo, 2

Carlos San Miguel Méndez,

Arturo Cruz Cidoncha,

Alvaro Robin Valle de Lersundi,

1 Department of Surgery, Henares University Hospital, Francisco de Vitoria University, Avenida de Marie Curie, s/n, 28822 Coslada, Madrid, Spain

2 Department of Surgery, Puerta de Hierro University Hospital, Madrid, Spain

3 Department of Surgery, La Fe University Hospital, Valencia, Spain

4 Department of Surgery, Monaldi Azienda Ospedaliera dei 


\section{Abstract}

\section{Background}

Optimal mesh reinforcement for abdominal wall reconstruction (AWR) in complex hernias remains questionable. Use of biologic, absorbable and synthetic meshes has been described. The idea of using an absorbable mesh (AM) under a permanent mesh (PM) in a retromuscular position may help in these challenging situations.

AQ1

\section{Methods}

Between 2011 and 2016, consecutive patients undergoing open AWR utilizing an AM as posterior layer reinforcement and configuration of a large PM were identified in a multicenter that prospectively maintained database in four hospitals. Main outcomes included demographics, ventral hernia classifications, perioperative data, complications and recurrences.

\section{Results}

A total of 169 complex incisional hernias were analyzed. Mean age was 60.9, with mean body mass index 30.7 (range: 20-46). Location of incisional hernias (IH) was: 80 midline, 59 lateral and 30 midline and lateral. $78 \%$ were grade I and II in Ventral Hernia Working Group classification. 52\% of patients were discharged with no complication. There were 19\% seromas, $13 \%$ hematomas, $12 \%$ surgical-site infection and 10\% skin dehiscence. Only partial mesh removal was necessary in one patient. After a mean follow-up of 26 months (range 15-59), there were five (3.2\%) recurrences. Reoperations on patients showed a band of fibrosis separating the peritoneum from the PM.

\section{Conclusion}


The combination of AM with very large PM in the same retromuscular position in AWR seems to be safe. The efficacy with recurrence rates below $4 \%$ in complex midline and lateral IH may be explained by the use of larger PMs that are extended and configured with the support of AMs. Reoperations on patients have confirmed the previous experimental reports on the use of the AM.

\section{Electronic supplementary material}

The online version of this article (https://doi.org/10.1007/s00268-018-4765-9) contains supplementary material, which is available to authorized users.

\section{Introduction}

Incisional hernia $(\mathrm{IH})$ repair is one of the most frequent operations performed in the world [1]. Some of these IH are considered to be complex, due to technical factors or the patient's characteristics [2]. In the last decade, there has been an important contribution for improvement in the surgical treatment of complex IH: the development of new retromuscular surgical techniques in AWR and the appearance of new meshes that reinforce the hernia repair in complex scenarios.

Although the established retromuscular preperitoneal dissection according to Rives-Stoppa may solve a number of these complex IHs [3, 4], the introduction of posterior components separation has facilitated the closure of both posterior and anterior layers in wide defects and the location of large meshes in a completely retromuscular preperitoneal space $[5,6,7,8]$. Transversus abdominis release (TAR) is the posterior components separation technique that has gained more popularity, and there are many publications regarding the successful treatment in a wide variety of complex repairs: multirecurrences, loss of domain, open abdomen and iliac hernias $[9,10,11,12]$.

To date, the meshes used after a TAR to reinforce the posterior layer have been synthetic, biologic and absorbable $[9,13,14]$. In the TAR technique, the openings on the peritoneum during the dissection are very common, and in very large defects or multirecurrent IHs, complete closure of posterior layer may be impossible. Under these surgical situations, the possibility of using a 
combination of an absorbable mesh (AM) with a permanent synthetic mesh (PM) as a barrier to separate the PM from viscera has been proposed [15]. Some of the potential benefits of the AM would be the complete closure of the posterior layer and the prevention of future adhesions of the bowel to the PM [16]. In 2011, our group started using AM to close the posterior layer in those cases of IH after urologic procedures in which the absence of peritoneum in Retzius space was a frequent finding. We realized that this AM not only avoided the contact of PM with the viscera but also provided a mechanical support to the extension and three-dimensional (3D) configuration of the PM, and started using both meshes in AWR. This idea was adopted by other European hospitals dedicated to complex IHs. The aim of this study is to present the results of the first multicenter experience using the combination of $\mathrm{AM}$ and PM in AWR.

\section{Methods}

From December 2011 to December 2016, patients with complex IH undergoing retromuscular repair with the combination of an absorbable and permanent meshes with a minimum follow-up of 15 months in four European institutions were identified in a prospectively maintained database. The hospitals involved in the study are recognized referral centers for complex abdominal wall repair. All the patients met the inclusion criteria to consider the IH to be complex [2]. We have included incisional hernias in midline (M1-M5 EHS classification) and lateral abdominal wall (L1-L4 EHS classification).

Patient demographics included age, sex, body mass index (BMI), comorbidities, the number of previous hernia operations and cause of first operation. Hernia characteristics included location, Carolina's risk index [17], length and width of defect and mesh area. Intraoperative wound assessments were recorded using Center for Disease Control (CDC) guidelines, and hernia grades were determined according to several classifications $[18,19,20]$. All patients had a preoperative computed tomography (CT) scan to evaluate the surgical strategy.

Postoperative data included both local and systemic complications, time in intensive care unit and length of hospitalization. Surgical-site events (SSE) include surgical-site infections (SSI) [21] and any wound dehiscence, seroma, skin necrosis or hematoma [19]. 
Standard follow-up protocol consisted of physical examination during a visit to the outpatient clinic at 6 weeks, 3 months, 6 months and every year. CT scans were routinely obtained in case of any abdominal discomfort or any doubt of potential recurrence on clinical exploration. Recurrence was defined as a recurrent hernia seen on CT scan or noted on physical examination by the primary surgeon. Bulging was defined as an area of weakness or asymmetry in the inspection or exploration of abdominal wall, but in the CT, there is no defect on the abdominal wall. Preoperative pneumoperitoneum was performed in 26 cases of loss of domain as it has been described [22].

\section{Surgical technique}

For midline incisional hernia (M1-M5 EHS classification), the repair was made through previous incisional scars. Previous implanted meshes were only removed in case of infection, fistula, lack of integration or intense adhesions. The sac was preserved until the moment when the closure of layers started. Typically, the sac is cut longitudinally to obtain two halves. One half of the sac was left attached to the posterior rectus sheath to facilitate posterior layer closure, and the contralateral flap of sac was used to cover the mesh in case of impossibility to approximate the midline. Adhesions of abdominal viscera to the abdominal wall were released. Then, a retrorectus dissection was performed in all cases according to Rives-Stoppa technique, preserving the neurovascular bundles that come to innervate the rectus muscle [23, 24]. When the midline could not be completely approximated despite the retrorectus dissection, a TAR was added. Briefly, the TAR was made down-to-up, starting with the lateral incision on the posterior rectus sheath from arcuate line, $1-\mathrm{cm}$ medial to linea semilunaris. Then, the preperitoneal plane was dissected from lateral to medial ascending the lateral incision on the posterior rectus sheath parallel to linea semilunaris to reach the fibers of transversus abdominis in epigastric area [25]. After cutting the fibers of the muscle as originally described [8], the preperitoneal plane was changed to the pre-transversalis plane. Then, the retromuscular plane behind the visceral sac is widely dissected as far as the central tendon of the diaphragm (cranially), Cooper's ligament (caudally) and psoas muscle (laterally to the tevel of posterior axillary line). In one case, a Carbonell technique was performed [5]. Then, in most cases, the posterior layer composed of the medialized posterior rectus sheaths and peritoneal sac could be closed with running absorbable monofilament sutures. The unavoidable tears 
greater than $1 \mathrm{~cm}$ on the peritoneum were also closed.

AQ2

Next, an absorbable $20 \times 30 \mathrm{~cm}$ mesh $\left(\mathrm{GORE}^{\circledR}\right.$ BIO-A ${ }^{\circledR}$ Tissue Reinforcement, W. L. Gore \& Associates, Inc. Flagstaff, AZ, USA), usually trimmed to adapt to the shape of the space dissected, was placed without any fixation to reinforce the posterior layer (Video 1 of Electronic Supplementary Material). At the myopectineal area, the mesh is usually cut to adjust to the shape of the ileopubic tracts. This mesh will help to make the "taco" or 3D configuration of the PM that will be shaped as Stoppa described [23] (Figs. 1 and 2) (Video 2 of Electronic Supplementary Material). A large uncoated macroporous PM was also placed in the same retromuscular position over the AM. The size of PMs used was $26 \times 36 \mathrm{~cm}$ polypropylene (Optilene mesh, B. Braun, Melsungen, Hessen, Germany) for Rives procedures, and $50 \times 50 \mathrm{~cm}$ polypropylene $\left(\right.$ Bulevb $^{\circledR}$, Dipro Medical Devices SRL, Torino, Italy) or $60 \times 45 \mathrm{~cm}$ polyvinylidene fluoride (PVDF) $\left(\right.$ Cicat $^{\circledR}$, Dynamesh, FEG Textiltechnik mbH Aachen, Germany) for TAR surgeries. These meshes were also cut to adjust to the size of the plane dissected (Figs. 1 and 2). These PMs were only secured cranially and caudally in the midline with slowly absorbable sutures: Cranially, retroxyphoid to the posterior rectus sheaths insertions on cartilages or to the central tendon of diaphragm. Caudally was only fixed with one 1 stitch to both Cooper's ligaments. No lateral or transparietal fixation was made. Drains were normally placed between the polypropylene mesh and the muscles. The linea alba was restored with slowly absorbable sutures. When the linea alba (the medial borders of the anterior rectus sheaths) could not be completely closed, the borders of the anterior rectus sheaths were fixed to both meshes, leaving a bridge that is usually covered with remnant of sacs or fibrous tissues. The maximum width of the bridge was registered. We have also considered bridging those cases when the anterior layer was completely closed with rest of fibrosis, previous meshes or peritoneal sac but not with anterior rectus sheaths. The redundant sacs, soft tissues and skins were then removed.

\section{Fig. 1}

a Shape of the PM in the Rives-Stoppa repair in midline IH. The arrow shows bending of the mesh at the retroinguinal area. b Shape of the AM to support the 3D configuration of PM in the Rives-Stoppa repair in midline IH. c PM placed over the AM to wrap inferiorly the visceral sac in the Rives-Stoppa repair in 


\section{midline IH}

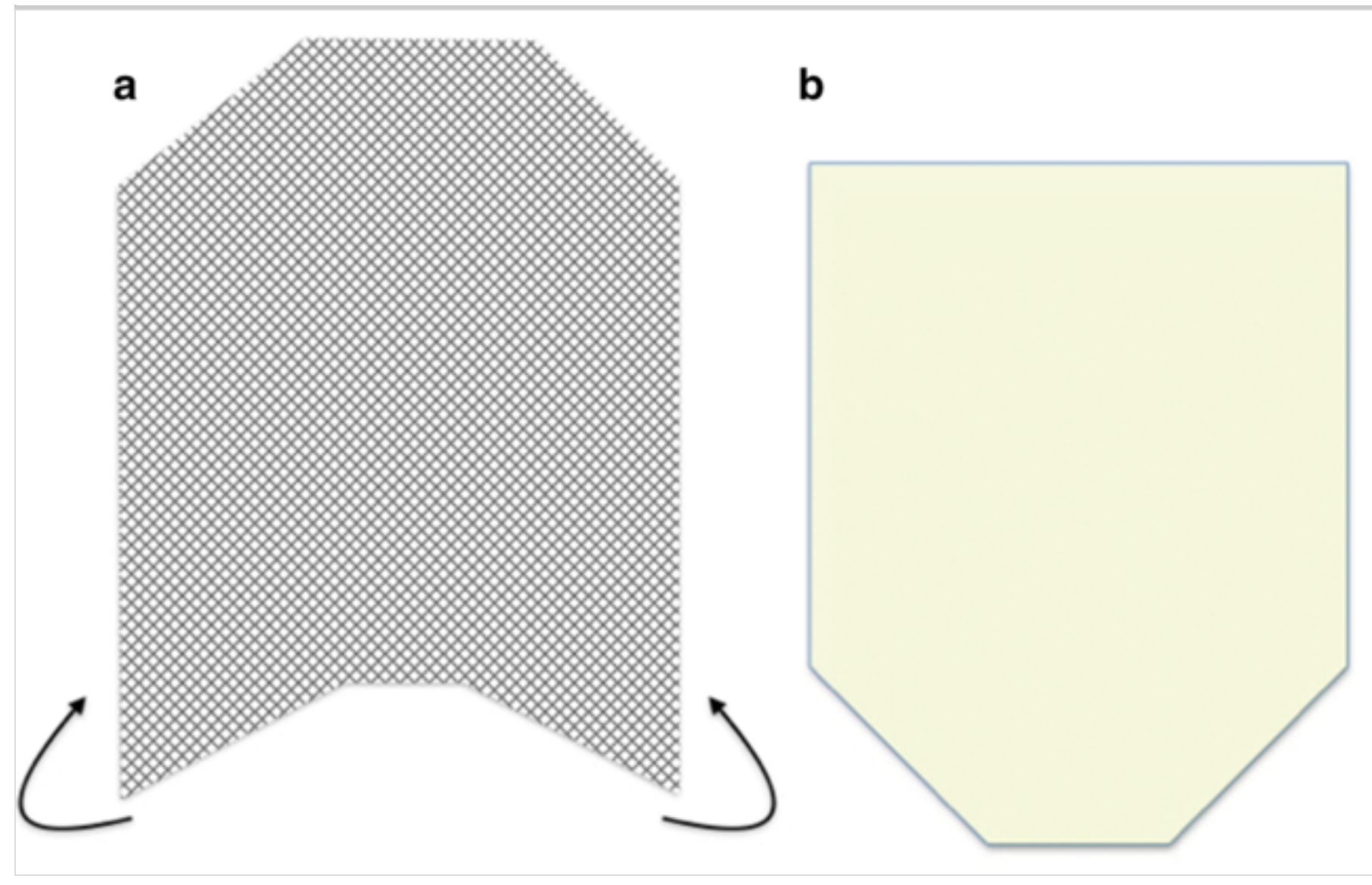

\section{Fig. 2}

a. Shape of the PM in the TAR repair in midline IH. Arrows 1 shows bending of the mesh at the retroinguinal area and arrows 2 shows bending of the mesh laterally toward the back over the psoas muscle, quadratus lumborum and diaphragm. b Shape of the absorbable mesh to support the $3 \mathrm{D}$ configuration of permanent mesh in TAR repair in midline IH 


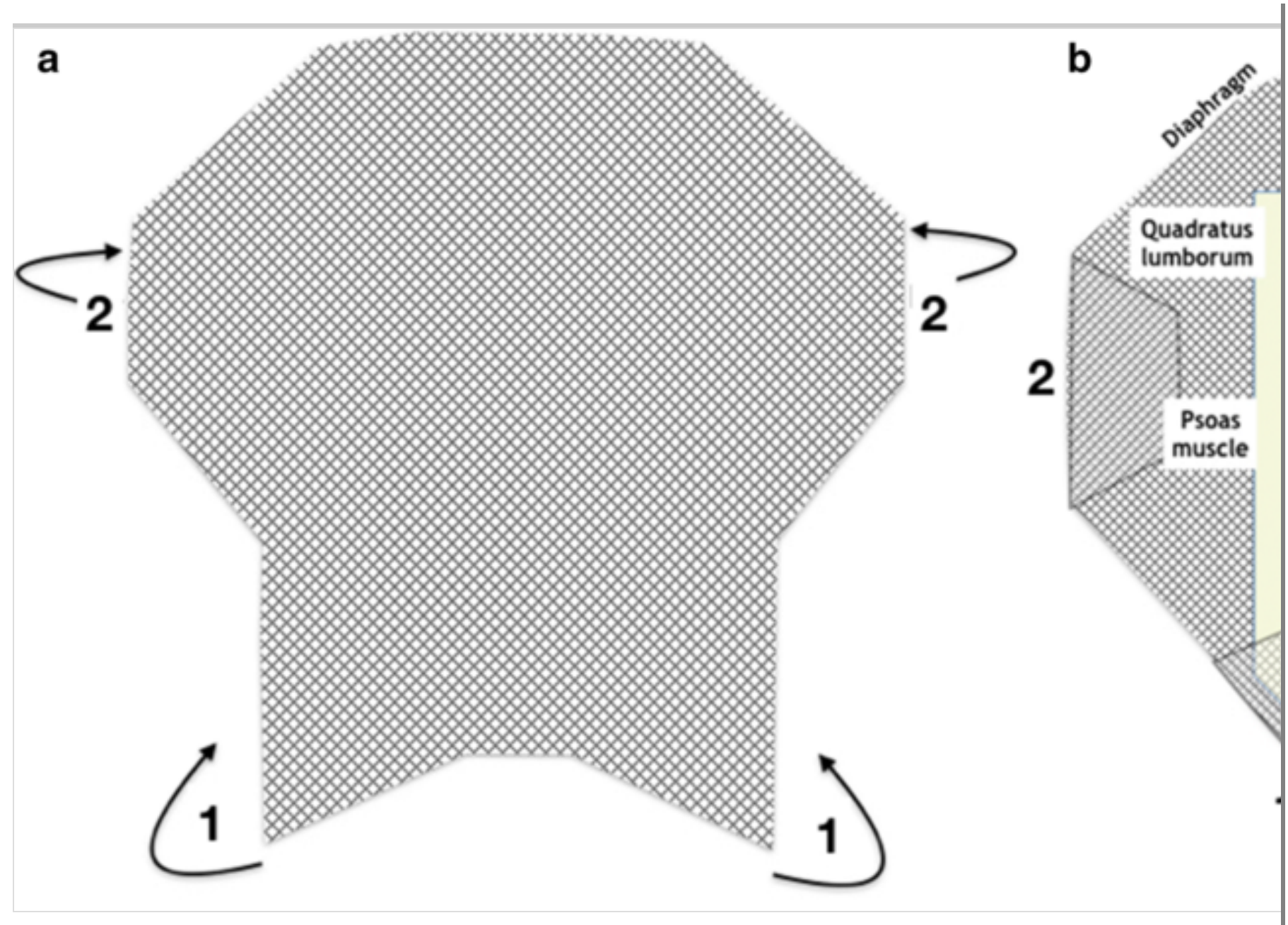

AQ3

AQ4

In lateral incisional hernias (L1-L4 EHS classification), a retromuscular preperitoneal dissection was made to achieve, at least, $10 \mathrm{~cm}$ dissection in all directions from the limits of the defect. When this dissection could not be enough to obtain adequate overlap of $10 \mathrm{~cm}$, then a TAR from lateral to medial was performed to obtain a wide preperitoneal retromuscular space. A reinforcement of AM and PM was also used in a similar way to midline $\mathrm{IH}$. The AM was placed again without fixation, and the PM was secured cranially with one or two transparietal stitches in the intercostal spaces and inferiorly to iliac crest and/or Cooper's ligaments. In most cases, the layers of lateral muscles could be closed with $2-3$ rows of running sutures. A drain was also regularly placed.

\section{Postoperative care}


Patients were extubated within the next $24 \mathrm{~h}$. Intradural catheters were used to control immediate postoperative pain but without following a strict protocol. Patients were discharged from the intensive care unit to the ward in 24-48 h, beginning oral intake if no clinical signs of ileus were present. Ambulation was also encouraged as soon as the patient arrived at the ward. Drains were removed when output was less than $50 \mathrm{~mL}$.

\section{Statistics}

To report the results of this cohort study, we have followed current recommendations [26, 27]. Quantitative variables were expressed as mean and standard deviation, and categorical variables, as absolute numbers and percentages. The comparative analysis was performed with the Student's $t$ test or the $\chi^{2}$ test. In the logistic regression analysis, we included those factors with $p<0.05$ in the univariate analysis. Overall recurrence as a function of time has been estimated using the Kaplan-Meier method. All statistical analysis was performed using SPSS v.18.0 (IBM Corp., Armonk, NY, USA).

\section{Results}

Our review of our prospective European database found 169 patients who met the inclusion criteria. We have operated more males than females (99/70), and mean age was 60.9. In Table 1, the demographics, comorbidities and characteristics of IH have been collected. Only three cases were operated in emergency situation. The different IH classifications that have been used are shown in Table 2. Regarding the location of IH, 80 (47.3\%) were middle IH, 59 (34.9\%) were lateral IH and 30 (17.8\%) were midline IH associated to a lateral IH. In eight patients (4.7\%), an inguinal hernia was discovered during preperitoneal dissection. In another two patients, a small lateral IH was also found, that was not previously diagnosed, probably due to drains implanted in the previous procedures.

\section{Table 1}

Patient demographics and comorbidities 


\begin{tabular}{|l|l|}
\hline Male & $99(58.6 \%)$ \\
\hline Female & $70(41.4 \%)$ \\
\hline Age, mean (range) & $60.9(32-86)$ \\
\hline BMI, mean (range) & $30.7(20.3-46.9)$ \\
\hline Obesity (BMI > 30) & $108(63.9 \%)$ \\
\hline Comorbidities & \\
\hline Smoking & $61(36.1 \%)$ \\
\hline Anticoagulation & $42(24.9 \%)$ \\
\hline Diabetes & $52(30.8 \%)$ \\
\hline Immunosuppression & $19(11.2 \%)$ \\
\hline COPD & $27(16.0 \%)$ \\
\hline Hypertension & $92(54.4 \%)$ \\
\hline Neoplasia & $65(38.5 \%)$ \\
\hline CEDAR mean (range) & $29.2(7-90)$ \\
\hline$<30 \%$ & $96(56.8 \%)$ \\
\hline $30-60 \%$ & $61(36.0 \%)$ \\
\hline$>60 \%$ & $12(7.1 \%)$ \\
\hline ASA, median & 2 \\
\hline I & $22(13.0 \%)$ \\
\hline II & $94(55.6 \%)$ \\
\hline III & $49(29.0 \%)$ \\
\hline IV & $4(2.4 \%)$ \\
\hline Prior history of hernias & $63(37.3 \%)$ \\
\hline Number of previous hernia repairs, mean (range) & $2.2(1-18)$ \\
\hline Time of previous surgery (years), mean (range) & $4.0 .5-25)$ \\
\hline Cause of first surgery & \\
\hline Digestive & \\
\hline Urologic & \\
\hline
\end{tabular}




\begin{tabular}{|l|l|}
\hline Liver-pancreatic & $24(14.2 \%)$ \\
\hline Abdominal wall & $21(12.4 \%)$ \\
\hline Gynecologic & $12(7.1 \%)$ \\
\hline Post-traumatic & $9(5.3 \%)$ \\
\hline Cardiac & $3(1.8 \%)$ \\
\hline Others & $3(1.8 \%)$ \\
\hline
\end{tabular}

ASA, American Society of Anesthesiologists physical status classification; BMI, body mass index in $\mathrm{kg} / \mathrm{m}^{2}$

\section{Table 2}

Characteristics of $\mathrm{IH}$

\section{Variables}

EHS classification

\begin{tabular}{|l|l|}
\hline Medial & $104(47.3 \%)$ \\
\hline M1-M3 & $9(5.3 \%)$ \\
\hline M1-M5 & $83(49.1 \%)$ \\
\hline M3-M5 & $12(7.1 \%)$ \\
\hline Lateral & $65(34.9 \%)$ \\
\hline L1 & $18(10.7 \%)$ \\
\hline L3 & $32(18.9 \%)$ \\
\hline L4 & $15(8.9 \%)$ \\
\hline
\end{tabular}

Slater's classification [2]

\begin{tabular}{|l|l|}
\hline Grade 1 & $9(5.3 \%)$ \\
\hline Grade 2 & $52(30.7 \%)$ \\
\hline Grade 3 & $108(63.9 \%)$
\end{tabular}

VHWG classification [19]

Grade 1

Grade 2

\section{Total patients $(n=169)$}




\begin{tabular}{|l|l|}
\hline Grade 3 & $29(17.2 \%)$ \\
\hline Grade 4 & $7(4.1 \%)$ \\
\hline Wound classification & $143(84.6 \%)$ \\
\hline Clean & $11(6.5 \%)$ \\
\hline Clean-contaminated & $11(6.5 \%)$ \\
\hline Contaminated & $4(2.4 \%)$ \\
\hline Dirty & \\
\hline VHSS [20] & $66(39.1 \%)$ \\
\hline Grade 1 & $77(45.6 \%)$ \\
\hline Grade 2 & $26(5.4 \%)$ \\
\hline Grade 3 & \\
\hline
\end{tabular}

VHWG, ventral hernia working group hernia classification; VHSS, ventral hernia staging system classification

Operative variables are shown in Table 3. Fourteen patients with midline IH could be solved with a Rives-Stoppa. The rest needed a posterior components separation technique. In eight patients (4.7\%), the posterior layer was reconstructed with the AM, suturing the mesh as a bridge to the peritoneum. A right-extended colectomy was used only in two cases when size of the visceral content made it impossible to close the posterior layer. In 39\%, the anterior layer was not completely closed with anterior rectus sheath, leaving an average bridge of $5 \mathrm{~cm}$ (range: 2-20) (Fig. 3). Mean time until for drain removal wa $10.3 \mathrm{~s} 10,3$ (range: $2-50$ ).

\section{Table 3}

Operative data

\section{Variables}

Total patients $(n=169)$

Type of surgery

\begin{tabular}{|l|l|}
\hline Elective & $166(98.2 \%)$ \\
\hline Emergency & $3(1.8 \%)$ \\
\hline
\end{tabular}

Size of defect in anterior layer 


\begin{tabular}{|l|l|}
\hline Width, cm, mean (range) & $12.7(4-40)$ \\
\hline Area, $\mathrm{cm}^{2}$, mean (range) & $447.3(240-1380)$ \\
\hline Surgical technique & \\
\hline Midline & $14(8.3 \%)$ \\
\hline Rives-Stoppa [23] & $1(0.6 \%)$ \\
\hline Carbonell [5] & $89(52.6 \%)$ \\
\hline TAR [6] & \\
\hline Lateral & $16(9.4 \%)$ \\
\hline Retromuscular preperitoneal [36] & $2(1.2 \%)$ \\
\hline Carbonell [5] & $47(27.8 \%)$ \\
\hline Reverse TAR [33] & $8(4.7 \%)$ \\
\hline Bridging of posterior layer after TAR* & 5 \\
\hline Midline & 3 \\
\hline Lateral & $66(39.0 \%)$ \\
\hline Bridging of anterior layer after TAR** & 47 \\
\hline Midline & $22(13.0 \%)$ \\
\hline Lateral & 19 \\
\hline Diameter of bridging after TAR, median (range) & $4(2-20) \mathrm{cm}$ \\
\hline $0-5$ cm & $41(62.1 \%)$ \\
\hline $5-10 \mathrm{~cm}$ & $20(30.3 \%)$ \\
\hline 10 cm & $5(7.6 \%)$ \\
\hline Associated surgery to the IH repair & $9(5.3 \%)$ \\
\hline Resection of omentum & $95.3 \%)$ \\
\hline Closure of bowel opening & \\
\hline Intestinal resection & \\
\hline Another abdominal surgery & \\
\hline Panniculectomy & \\
\hline & \\
\hline
\end{tabular}


Size of mesh used, $\mathrm{cm}^{2}$

\begin{tabular}{|l|l|}
\hline Absorbable & $555(300-1200)$ \\
\hline Permanent & $2100(750-3000)$ \\
\hline \begin{tabular}{l} 
Type of permanent mesh used \\
\hline Polypropylene
\end{tabular} & $143(84.6 \%)$ \\
\hline PVDF & $26(15.4 \%)$ \\
\hline Operative time, mean (range) & $219(65-490)$ min \\
\hline TAR, transversus abdominis release; PVDF, polyvinylidene fluoride \\
\hline$*$ Impossibility to completely close peritoneum and/or posterior rectus sheaths \\
\hline$* *$ Impossibility to completely close linea alba (borders of anterior rectus sheaths) \\
\hline
\end{tabular}

Fig. 3

Histogram of the size of the bridge of patients in whom it was not possible to completely close the anterior rectus sheaths in the midline

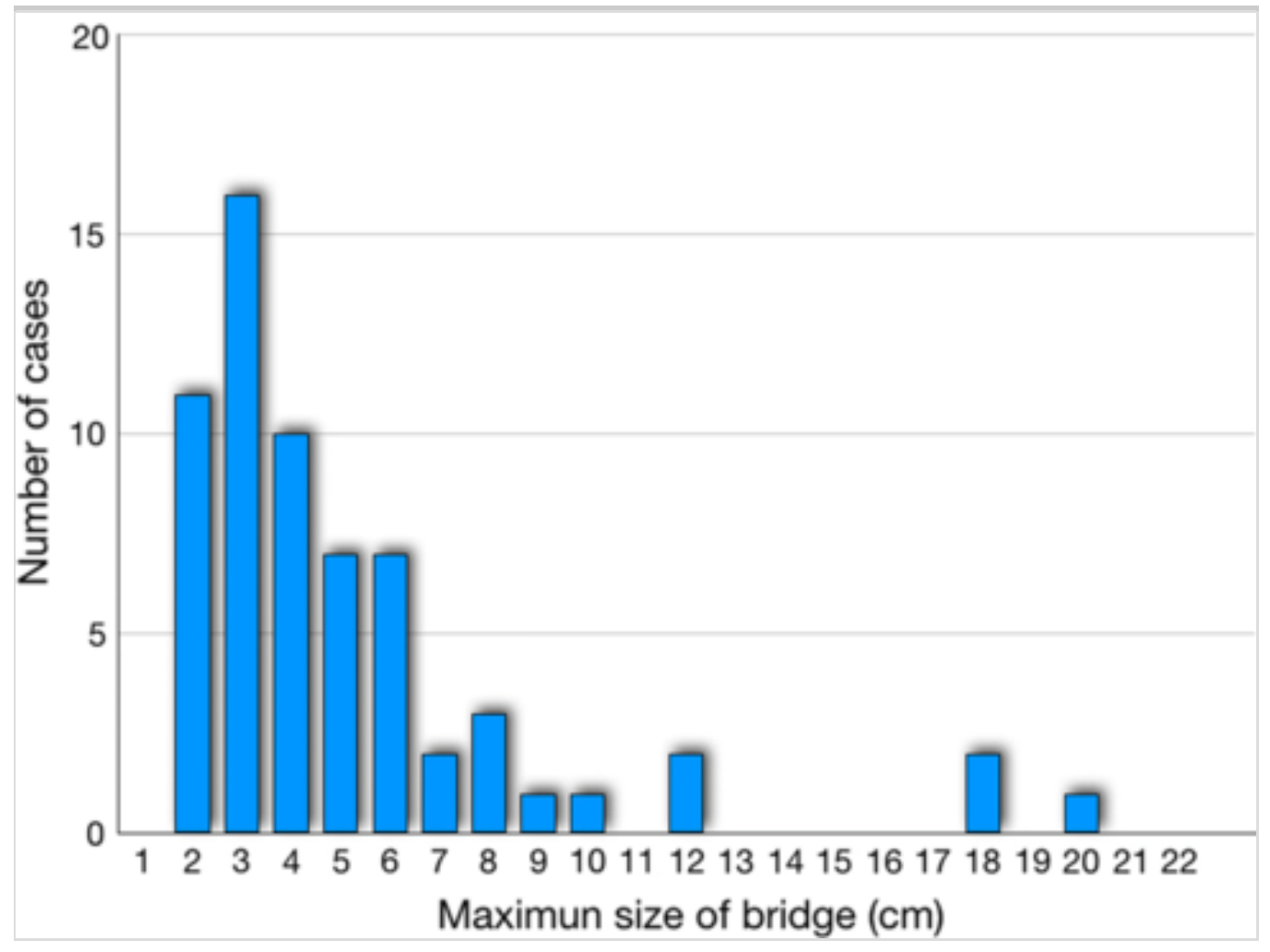


The complications observed are shown in Table 4 and classified according to Clavien-Dindo classification [28]. 60.4\% of patients were discharged without any surgical-site event. $12.4 \%$ developed a SSI. There was no SSI in lateral incisional hernias. Table 5 shows the perioperative factors statistically significant in the univariate analysis. In the multivariate analysis, wound classification was an independent factor for SSI, ileus and respiratory insufficiency ( $p=0.007,0.016$ and 0.009 ). VHSS classification was also an independent risk factor for seroma $(p=0.013)$ and panniculectomy for skin dehiscence (0.02).

\section{Table 4}

Postoperative complications

\begin{tabular}{|c|c|c|}
\hline & $N(\%)$ & Clavien-Dindo $>1$ \\
\hline Any complication & $81(47.9 \%)$ & \\
\hline \multicolumn{3}{|l|}{ Surgical-site events } \\
\hline Any surgical-site event & $67(39.6 \%)$ & \\
\hline Surgical-site infection & $21(12.4 \%)$ & \\
\hline Superficial & $15(8.9 \%)$ & $1 \mathrm{IIIb}$ \\
\hline Deep & $4(2.4 \%)$ & 1 IIIa \\
\hline Organ/space & $2(1.2 \%)$ & $2 \mathrm{IIIb}$ \\
\hline Hematoma & $23(13.6 \%)$ & 1 IIIa, 2 IIIb \\
\hline Seroma & $33(19.5 \%)$ & \\
\hline Skin/wound dehiscence & $17(10.1 \%)$ & \\
\hline \multicolumn{3}{|l|}{ Abdominal complications } \\
\hline Paralytic ileus & $13(7.7 \%)$ & 9 II \\
\hline Intestinal fistula & $4(2.4 \%)$ & 3 IIIa, 1 V \\
\hline Intra-abdominal hypertension & $2(1.2 \%)$ & $2 \mathrm{IVa}$ \\
\hline \multicolumn{3}{|l|}{ Systemic complications } \\
\hline Urinary infection & $4(2.4 \%)$ & \\
\hline Venous line infection & $4(2.4 \%)$ & \\
\hline
\end{tabular}




\begin{tabular}{|l|l|l|}
\hline Respiratory insufficiency & $8(4.7 \%)$ & 6 II, 2 IVa \\
\hline Renal insufficiency & $3(1.7 \%)$ & 3 II \\
\hline Pneumonia & $2(1.2 \%)$ & 2 II \\
\hline Cardiac complications & $4(2.4 \%)$ & 3 III, 1 V \\
\hline DVT/PE & $1(0.5 \%)$ & 1 II \\
\hline Clostridium difficile sepsis & $1(0.5 \%)$ & 1 V \\
\hline Pain $>48$ h requiring opioids & $28(16.6 \%)$ & \\
\hline ICU stay & $79(46.7 \%)$ & \\
\hline Mean (range) & $2.4(1-14)$ & \\
\hline Length of hospitalization, mean (range) & $8.7(3-98)$ & \\
\hline Mortality & $3(1.7 \%)$ & \\
\hline Readmission & $15(8.9 \%)$ & \\
\hline $\begin{array}{l}\text { DVT/PE, deep venous thrombosis/pulmonary } \\
\text { care unit }\end{array}$ & & \\
\hline
\end{tabular}

\section{Table 5}

Statistically significant perioperative variables for postoperative complications

\begin{tabular}{|l|l|l} 
Complication & $p$ & OR $(\mathbf{C I}) *$ \\
\hline SSI & &
\end{tabular}

\begin{tabular}{|l|l|}
\hline Wound classification & 0.001 \\
\hline VHWG & 0.009 \\
\hline VHSS & 0.018 \\
\hline
\end{tabular}

\section{Seroma}

\begin{tabular}{|l|l|l|}
\hline Obesity $(\mathrm{BMI}>30)$ & 0.004 & $0.45(0.20-0.97)$ \\
\hline VHSS & 0.007 & \\
\hline Hematoma & & \\
\hline Hypertension & 0.014 & \\
\hline
\end{tabular}

Skin/wound dehiscence 


\begin{tabular}{|c|c|c|}
\hline Hypertension & 0.015 & $3.5(1.23-1.93)$ \\
\hline Immunosuppression & 0.001 & $11.3(3.67-35.37)$ \\
\hline ASA & 0.003 & \\
\hline Panniculectomy & 0.034 & $3.3(1.03-10.54)$ \\
\hline \multicolumn{3}{|c|}{ Respiratory insufficiency } \\
\hline ASA & 0.003 & \\
\hline COPD & 0.003 & $10.5(2.34-47.21)$ \\
\hline Wound classification & 0.002 & \\
\hline CEDAR & 0.01 & \\
\hline VHWG & 0.037 & \\
\hline VHSS & 0.001 & \\
\hline \multicolumn{3}{|l|}{ Neumonia } \\
\hline Wound classification & 0.004 & \\
\hline VHSS & 0.004 & \\
\hline \multicolumn{3}{|l|}{ Postoperative ileus } \\
\hline Hypertension & 0.004 & $11.4(1.44-89.80)$ \\
\hline Immunosuppression & 0.043 & $4.17(1.14-15.21)$ \\
\hline ASA & 0.018 & \\
\hline Wound classification & 0.001 & \\
\hline VHWG & 0.002 & \\
\hline VHSS & 0.007 & \\
\hline \multicolumn{3}{|l|}{ Cardiac complications } \\
\hline ASA & 0.018 & \\
\hline \multicolumn{3}{|c|}{$\begin{array}{l}\text { OR }(\mathrm{CI})^{*} \text {, odds ratio. Confidence interval, when applicable; VHWG, ventral } \\
\text { hernia working group classification; VHSS, ventral hernia staging system } \\
\text { classification; ASA, American Society of Anesthesiologists; COPD, chronic } \\
\text { obstructive pulmonary disease }\end{array}$} \\
\hline
\end{tabular}


Three patients died in the immediate postoperative period: 1 from sepsis due to anastomotic fistula, one from cardiac failure after emergency surgery and one from Clostridium difficile sepsis on postoperative day 3. None of the patients have developed an intraparietal hernia into the retrorectus space or preperitoneal space. Follow-up could be accomplished in the outpatient clinic in $91.7 \%$ of patients $(n=155)$. One patient was readmitted 7 weeks after surgery due to fever and abdominal pain. The CT scan revealed a localized inflammatory reaction on the right rectus muscle without collections to be drained, and the patient was discharged after 7 days of broad-spectrum antibiotic therapy.

Three patients died during surveillance from reasons not related to IH: a recurrent tumor disease, a stroke and a cardiac disease. With a mean follow-up of 26.4 months (range: 15-59), we have had identified five recurrences (3.2\%): one epigastric recurrence after a Rives-Stoppa; one central recurrence in a patient that had a postoperative fistula after a TAR that drained through the wound and the mesh was partially removed in the midline to control local sepsis; two patients with a symptomatic bulging in the midline after a bridged TAR; and one patient with a symptomatic bulging after a retromuscular repair in a lumbar hernia. The timing of recurrence was between 10 and 21 months (Fig. 4). The last four patients with recurrence have already been reoperated with a new retromuscular preperitoneal approach. In these reoperations, it was not difficult to enter the space between the fibrosis over the peritoneum caused by the AM and the fibrosis infiltrating the PM (Fig. 5). The pathologic specimens taken at reoperations showed a thick band of fibrosis replacing the AM and separating it from the fibrosis of the PM (Figs. 6 and 7). After 2 years, another patient underwent an emergency surgery due to intestinal obstruction by an adhesion of small bowel to pelvis. In this reoperation, we could also check the replacement by fibrous tissue of AM and a lack of adhesions to abdominal wall.

Fig. 4

Estimated freedom of incisional hernia curve (Kaplan-Meier) 


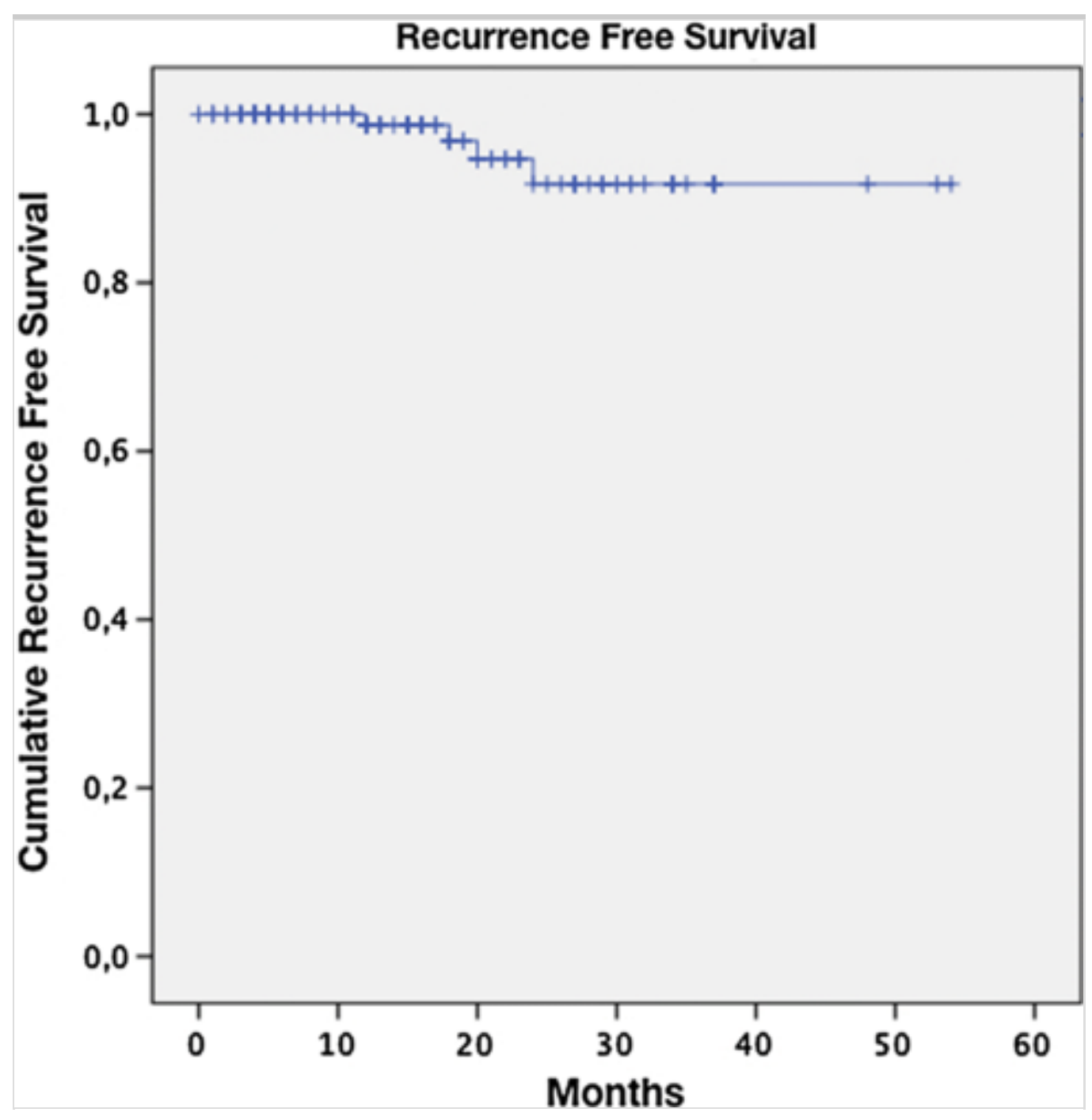

Fig. 5

Picture of the reoperation on one of the recurrent lumbar IH after the combination of absorbable and permanent mesh repair. The fibrosis infiltrating the permanent mesh (a) could be easily detached from the fibrosis over the peritoneum that have replaced the absorbable mesh (b) 


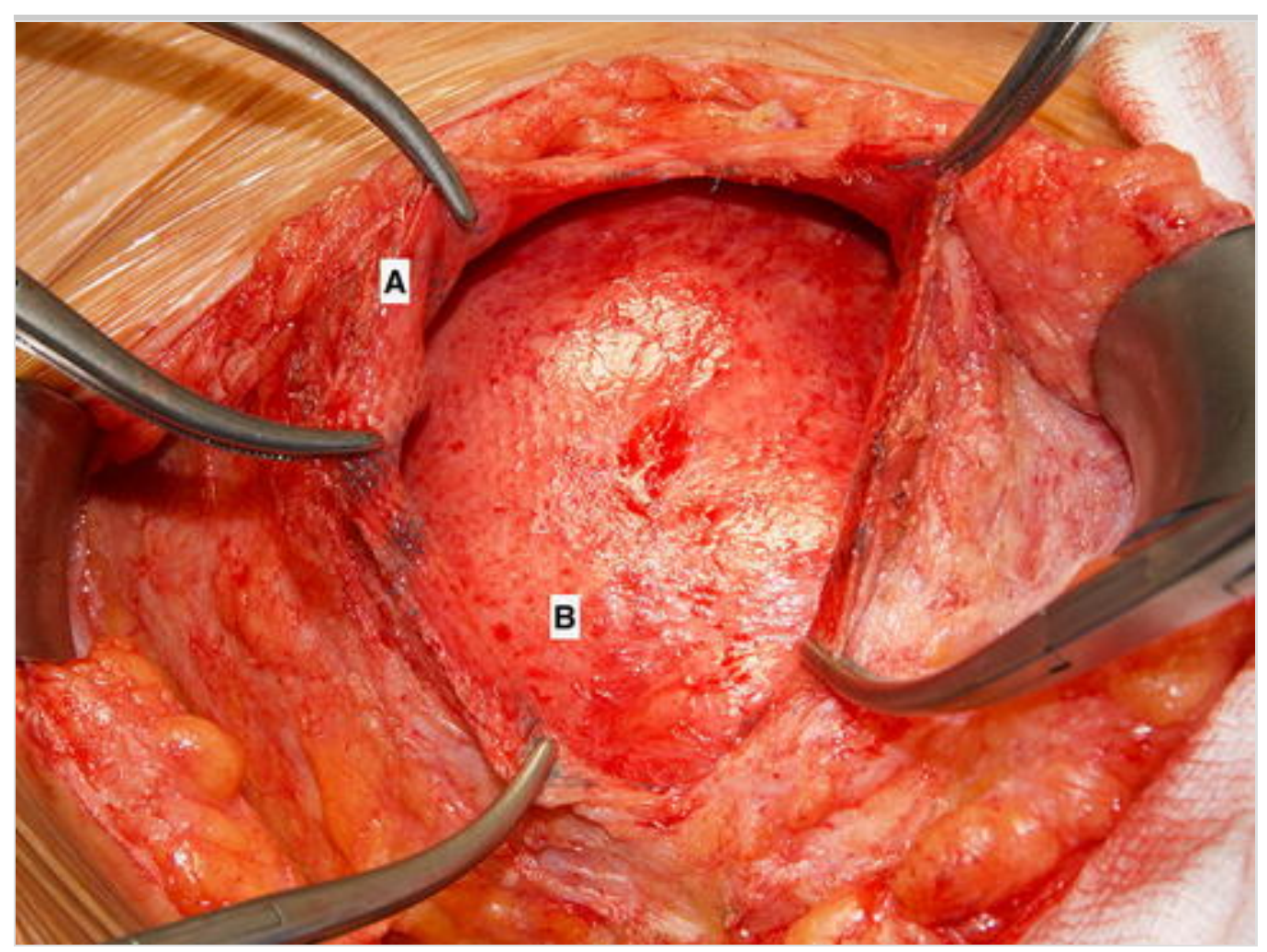

\section{Fig. 6}

Low-magnification image of representative pathologic examination of biopsy taken at reoperation of patient of Fig. 5. Layer A denotes areas where rare device remnants reside. Layer $\mathrm{B}$ is region of highly oriented and densely packed collagen. Layer $\mathrm{C}$ on the periphery is composed of loosely arranged collagen fibers. H\&E stain 


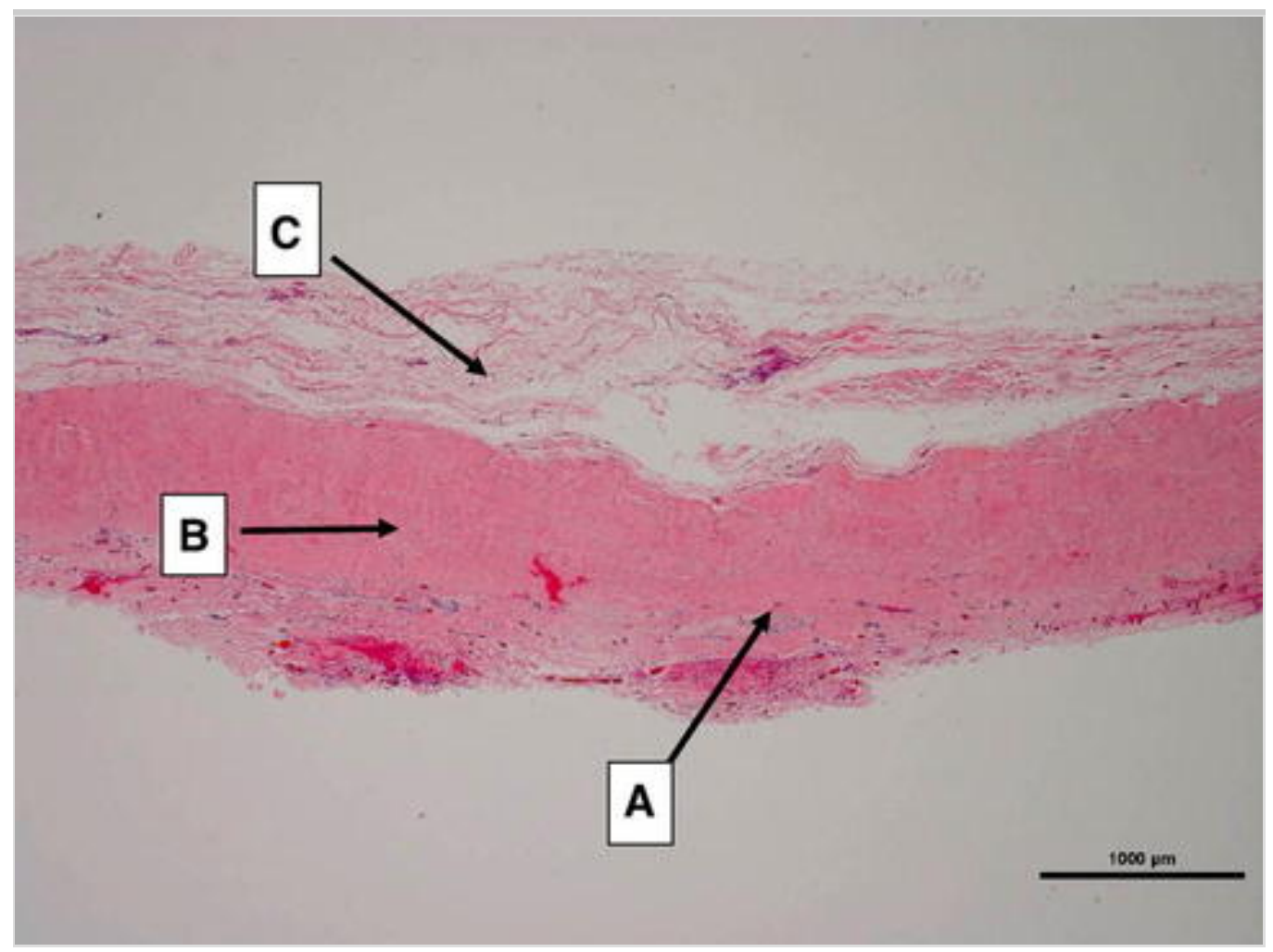

\section{Fig. 7}

High-magnification image of Fig. 6. Small number of macrophages with intracellular material (arrows) are present along the base of thick band of collagenous tissue. H\&E stain 


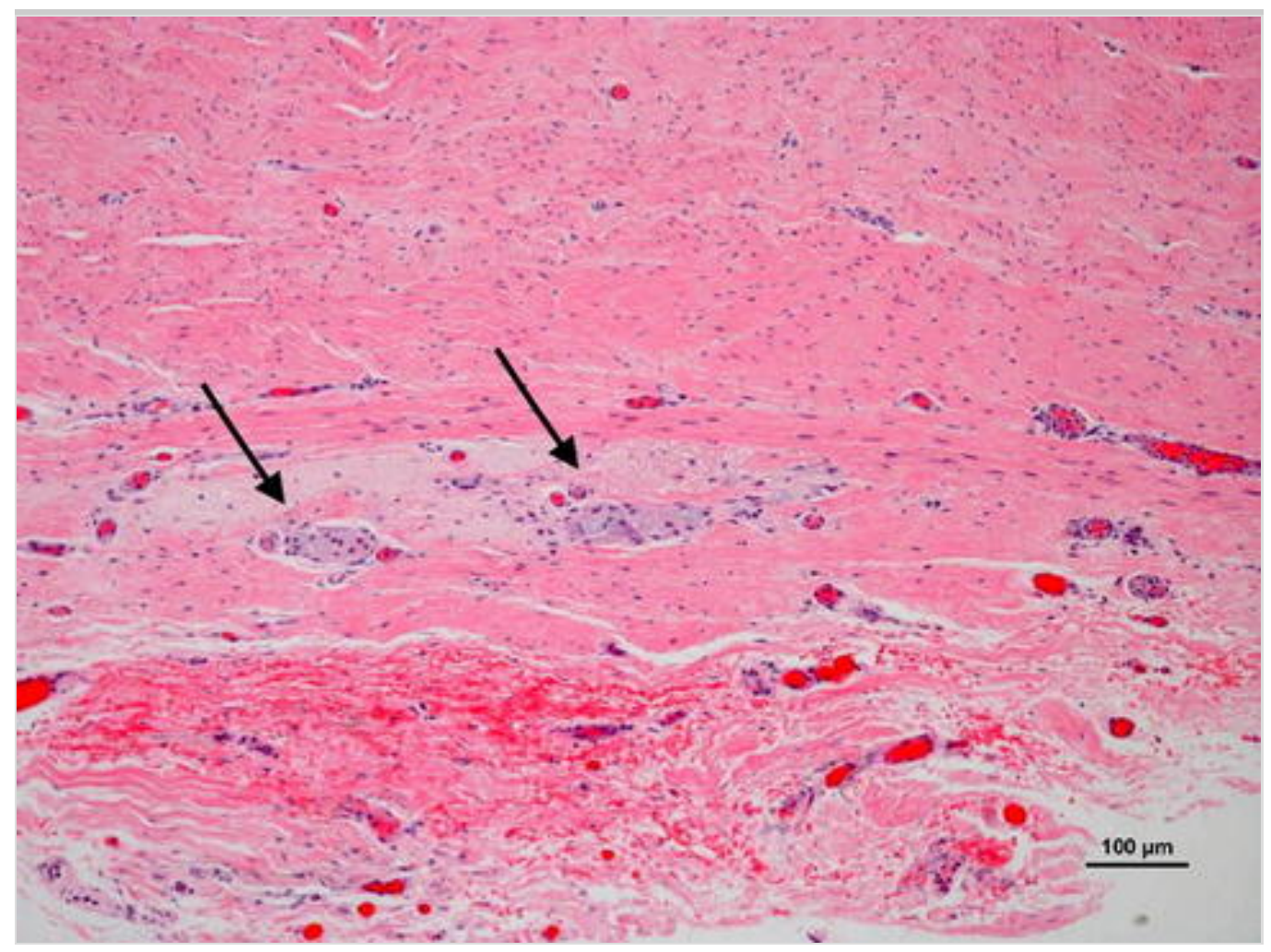

Regarding the other reported long-term complications, there are three patients with chronic pain, and four with chronic superficial seroma- two of them with reoperations after failure of conservative treatment. We have not observed any complication related to fistulae or chronic mesh infection. The only mesh that has been partially removed was that due to the postoperative fistula of anastomosis that had to be reoperated of recurrence.

\section{Discussion}

There have been several studies demonstrating that sublay mesh reinforcement after posterior components separation technique is safe and effective, with recurrence rates below $10 \%[9,10,11]$. In fact, in the largest cohort study published with 428 patients, the recurrence rate was $3.7 \%$ with a mean followup of more than 30 months [13]. Although our follow-up is not as long, we have observed even fewer recurrences $(n=5)$ during our clinical follow-up of more than $90 \%$ of patients included. This low rate is arguably the result of the retromuscular approach with posterior components technique and the combination of meshes used as sublay reinforcement. There are two significant aspects that suggest that the combination of meshes is advantageous for AWR. First, we have not observed in our series the central mesh recurrence due to 
breakdown of the mesh that has been observed in other studies using lowdensity meshes [29]. The only central recurrence was in a patient in whom the mesh was partially removed to control local sepsis. The second aspect is that the AWR can be bridged without producing high number of recurrences; $39 \%$ of patients have been bridged in our series (Table 3), and we have only seen five recurrences up to date. The elevated rate of incomplete closure of anterior layer may be related to the detail that we strictly define as incomplete, those patients with any final gap between the true medial borders of anterior rectus sheaths or anterior aponeurosis. Those patients in which the midline is finally closed with remnant of healing tissues, previous meshes, fibrosis or sacs are also considered as bridging. In the previous studies using biologic meshes, there were more recurrences and complications in those patients who underwent a bridged repair $[30,31,32]$. We have not observed these differences, possibly due to the fact that the combination of meshes implanted achieves a durable repair that was checked in the reoperated patients (Fig. 5). However, we strongly agree with the accepted recommendation to avoid bridging whenever possible as 2 out of 5 of our recurrences were in bridged repairs.

The posterior components separation technique allows the perfect application of the principles of "giant reinforcement of the visceral sac" proposed by Stoppa [23]. As it was initially described [6], and later thoroughly explained [8], the space obtained is extended from the diaphragm to Cooper's ligaments (craniocaudally) and from the quadratus lumborum and psoas muscles (transversely). In our experience, these principles are not only useful for midline but also for lateral defects that can be very difficult to repair due to the proximity of bones structures and the lack of aponeurosis outside the limit of the linea semilunaris. The extension of the retromuscular space between the parietal peritoneum and the muscles allows achieving a great overlap for subcostal, lumbar and iliac defects. This space can also be continued to the midline making a reverse TAR, from lateral to medial [33].

$52 \%$ of patients were discharged without any complication, both wound and systemic, despite the characteristics of the patients included. Very few patients had to be reoperated under general anesthesia due to specific wound complications: two hematomas and three SSI. Interestingly, hypertension was a risk factor for hematomas in the univariate analysis. The few but severe systemic complications observed reveal the complexity of these patients that 
had a mean CEDAR of $29 \%$. Although in our series CEDAR was not statistically significant associated to any specific complication, this mobile application has helped us in explaining to the patient the importance of controlling risk factors to reduce morbidity [17]. The comparative analysis demonstrated that the different classifications of complexity that have been used are valuable. In our series, the most recent VHSS predicted more postoperative complications. We agree that its simplicity may help in the stratification of morbidity [20]. Panniculectomy was associated with more skin dehiscence, confirming that adding this procedure increases the number of complications after IH open repair [34].

The use of an AM in combination with a PM was advocated in an experimental study [15]. Recently, a series of 36 patients from two different hospitals have been reported using an AM as a bridge on the posterior layer [16]. The AM used in these studies was made of short-term absorbable polyglactin. In the experimental study focused on the mesh-viscera interphase, the AM did not prevent adhesions formation but formed a thick fibrous capsule that seemed to protect the PM from the viscera. This experimental observation has been confirmed clinically in our reoperated patients, using a mid-term AM, made up of polyglycolic acid and trimethylene carbonate. We have also observed this band of fibrosis covering the peritoneum that has replaced the AM (Figs. 6 and 7). This finding also confirmed the experimental studies made on the host tissue response to this AM in which the mesh was gradually absorbed, served as tissue-building scaffold and replaced by a high-quality connective tissue [35]. No intense adhesions to the repaired abdominal wall were seen in any of the reoperated patients. This can be explained due to the placement of the AM in the retromuscular sublay position under the PM. Only in eight patients, the AM was used as a bridge to close the posterior layer, facing the viscera. In these clinical scenarios where the defect on posterior layer cannot be closed, we also agree that the combination of AM may protect the viscera from the synthetic uncoated mesh, and they are preferable to composite meshes that may produce seromas or infections [15]. Interestingly, the PM infiltrated by fibrosis could be easily peeled off from the fibrous tissue on the peritoneum, and the retromuscular space could be dissected again in these second-look operations (Fig. 5). In two cases, the peritoneum was not even opened during this repeated retromuscular dissection. We really think this is an additional advantage to use a 
combination of meshes in these complex situations.

Although the initial rigidity on manipulation of the AM may be awkward, we have found it very useful in locating the mesh in the sublay retromuscular preperitoneal position without any means of fixation (Video 1 and 2). It also covers the small holes in the peritoneum that are easily made during the TAR. Inferiorly, the mesh is trimmed to adapt to shape of both ileopubic tracts, allowing the 3D-configuration of the PM that was cut following the shape that Stoppa recommended [23] (Figs. 1 and 2). The rigidity of the AM acts like a cardboard and helps in extending the PM over it because, otherwise, it wrinkles and easily folds in such large dissected spaces of more than $1500 \mathrm{~cm}^{2}$. It is also helpful in lumbar and large subcostal incisional hernias because the convexity and extension of the PM are given by the rigidity of the AM. Furthermore, the its $1.71,7 \mathrm{~mm}$ thickness of the mesh allows suturing the borders of the fascial and muscular defects in case of bridging with no danger of injury to the viscera that would be only protected with a thin layer of peritoneum without this absorbable mesh [25].

AQ6

Nevertheless, our series presents important limitations. Although it is a prospective multicenter study, there is no group for comparison. So, we cannot conclude that the combination of AM with PM is better than utilizing the PM alone, although the operative findings in reoperated cases were very persuasive. While most recurrences appear before 24 months in most studies, longer followup is going to be necessary to confirm that any other long-term complication will not occur, like more recurrences, adhesions, fistulas or chronic infection. Finally, we have not included a quality-of-life study on our patients that would undoubtedly give more valuable results.

This multicenter series shows that the combination of AM with large PM in the same retromuscular position in AWR is safe up to a mean 2-year follow-up. The efficacy with recurrence rates below $4 \%$ in complex midline and lateral IH may be explained by the use of larger PMs placed in a retromuscular position that are shaped and extended with the support of absorbable meshes. These results are good despite the incomplete closure of the anterior layer and lack of lateral fixations of the meshes. The reoperations on patients have confirmed clinically that the AM used in this study is replaced by fibrous tissue that protects the 
viscera from the PM as observed in the previous experimental reports.

Compliance with ethical standards

Conflict of interest García-Urena has received speaker fees for symposium organized by Dynamesh, Braun, and Gore. Lopez-Monclús has received speaker fees for symposium organized by Gore.

\section{Electronic supplementary material}

Below is the link to the electronic supplementary material.

\section{VIDEO 1}

3D-configuration of a large PM with the support of AM after a left TAR and right Rives in a large iliac incisional hernia (MP4 55,539 kb)

\section{VIDEO 2}

Stoppa configuration and extension of a large PM after a bilateral TAR in a complex midline incisional hernia (MP4 28,417 kb)

\section{References}

1. Bower C, Roth JS (2013) Economics of abdominal wall reconstruction. Surg Clin North Am 93:1241-1253

2. Slater NJ, Montgomery A, Berrevoet F et al (2014) Criteria for definition of a complex abdominal wall hernia. Hernia 18:7-17

3. Iqbal CW, Pham TH, Joseph A, Mai J, Thompson GB, Sarr MG (2007) Long-term outcome of 254 complex incisional hernia repairs using the modified Rives-Stoppa technique. World J Surg 31:2398-2404. https://doi.org/10.1007/s00268-007-9260-7

4. Mehrabi M, Jangjoo A, Tavoosi H, Kahrom M, Kahrom H (2010) Long- 
term outcome of Rives-Stoppa technique in complex ventral incisional hernia repair. World J Surg 34:1696-1701. https://doi.org/10.1007/s00268010-0426-3

5. Carbonell AM, Cobb WS, Chen SM (2008) Posterior components separation during retromuscular hernia repair. Hernia 12:359-362

6. Novitsky YW, Elliott HL, Orenstein SB, Rosen MJ (2012) Transversus abdominis muscle release: a novel approach to posterior component separation during complex abdominal wall reconstruction. Am J Surg 204:709-716

7. Posielski NM, Yee ST, Majumder A, Orenstein SB, Prabhu AS, Novitsky YW (2015) Repair of massive ventral hernias with "quilted" mesh. Hernia 19:465-472

8. Gibreel W, Sarr MG, Rosen M, Novitsky Y (2016) Technical considerations in performing posterior component separation with transverse abdominis muscle release. Hernia 20:449-459

9. Pauli EM, Wang J, Petro CC, Juza RM, Novitsky YW, Rosen MJ (2015) Posterior component separation with transversus abdominis release successfully addresses recurrent ventral hernias following anterior component separation. Hernia 19:285-291

10. Winder JS, Behar BJ, Juza RM, Potochny J, Pauli EM (2016) Transversus abdominus release for abdominal wall reconstruction: early experience with a novel technique. J Am Coll Surg 223:271-278

11. Petro CC, Como JJ, Yee S, Prabhu AS, Novitsky YW, Rosen MJ (2015) Posterior component separation and transversus abdominis muscle release for complex incisional hernia repair in patients with a history of an open abdomen. J Trauma Acute Care Surg 78:422-429

12. Petro CC, Orenstein SB, Criss CN et al (2015) Transversus abdominis muscle release for repair of complex incisional hernias in kidney transplant recipients. Am J Surg 210:334-339 
13. Novitsky YW, Fayezizadeh M, Majumder A, Neupane R, Elliott HL, Orenstein SB (2016) Outcomes of posterior component separation with transversus abdominis muscle release and synthetic mesh sublay reinforcement. Ann Surg 264:226-232

14. Rosen MJ, Bauer JJ, Harmaty M et al (2017) Multicenter, prospective, longitudinal study of the recurrence, surgical site infection, and quality of life after contaminated ventral hernia repair using biosynthetic absorbable mesh: the COBRA study. Ann Surg 265:205-211

15. Liu L, Petro C, Majumder A, Fayezizadeh M, Anderson J, Novitsky YW (2016) The use of Vicryl mesh in a porcine model to assess its safety as an adjunct to posterior fascial closure during retromuscular mesh placement. Hernia 20:289-295

16. Winder JS, Majumder A, Fayezizadeh M, Novitsky YW, Pauli EM (2018) Outcomes of utilizing absorbable mesh as an adjunct to posterior sheath closure during complex posterior component separation. Hernia 22:303-309

17. Augenstein VA, Colavita PD, Wormer BA et al (2015) CeDAR: Carolinas equation for determining associated risks. J Am Coll Surg 221:S65-S66

18. Muysoms FE, Miserez M, Berrevoet F et al (2009) Classification of primary and incisional abdominal wall hernias. Hernia 13:407-414

19. Ventral Hernia Working G, Breuing K, Butler CE et al (2010) Incisional ventral hernias: review of the literature and recommendations regarding the grading and technique of repair. Surgery 148:544-558

20. Petro CC, O'Rourke CP, Posielski NM et al (2016) Designing a ventral hernia staging system. Hernia 20:111-117

21. Mangram AJ, Horan TC, Pearson ML, Silver LC, Jarvis WR, Guideline for Prevention of Surgical Site Infection (1999) Centers for Disease Control and Prevention (CDC) Hospital Infection Control Practices Advisory 
Committee. Am J Infect Control 1999(27):97-132

22. Bueno-Lledo J, Torregrosa A, Ballester N et al (2017) Preoperative progressive pneumoperitoneum and botulinum toxin type $\mathrm{A}$ in patients with large incisional hernia. Hernia 21:233-243

23. Stoppa RE (1989) The treatment of complicated groin and incisional hernias. World J Surg 13:545-554. https://doi.org/10.1007/BF01658869

24. Stoppa RE (2003) Wrapping the visceral sac into a bilateral mesh prosthesis in groin hernia repair. Hernia 7:2-12

25. Robin A, Blazquez Hernando L, López-Monclús J et al (2018) How we do it: down to up posterior components separation. Langenbecks Arch Surg 403(4):539-546

26. von Elm E, Altman DG, Egger M et al (2007) The strengthening the reporting of observational studies in epidemiology (STROBE) statement: guidelines for reporting observational studies. Ann Intern Med 147:573-577

27. Muysoms FE, Deerenberg EB, Peeters E et al (2013) Recommendations for reporting outcome results in abdominal wall repair: results of a consensus meeting in Palermo, Italy, 28-30 June 2012. Hernia 17:423-433

28. Clavien PA, Barkun J, de Oliveira ML et al (2009) The Clavien-Dindo classification of surgical complications: five-year experience. Ann Surg 250:187-196

29. Petro CC, Nahabet EH, Criss CN et al (2015) Central failures of lightweight monofilament polyester mesh causing hernia recurrence: a cautionary note. Hernia 19:155-159

30. Booth JH, Garvey PB, Baumann DP et al (2013) Primary fascial closure with mesh reinforcement is superior to bridged mesh repair for abdominal wall reconstruction. J Am Coll Surg 217:999-1009

31. Giordano S, Garvey PB, Baumann DP, Liu J, Butler CE (2017) Primary 
fascial closure with biologic mesh reinforcement results in lesser complication and recurrence rates than bridged biologic mesh repair for abdominal wall reconstruction: a propensity score analysis. Surgery 161:499-508

32. Garvey PB, Giordano SA, Baumann DP, Liu J, Butler CE (2017) Longterm outcomes after abdominal wall reconstruction with acellular dermal matrix. J Am Coll Surg 224:341-350

33. Garcia-Urena M, Lopez-Monclus J, Blazquez Hernando L, et al (2016) Giant bilateral subcostal incisional hernia solved by rectromuscular repair and transversus abdomins release. In: Ciné-Med, Inc. https://cinemed.com/acsonline/ Accessed 15 May 2018

34. Ross SW, Oommen B, Huntington C et al (2015) National outcomes for open ventral hernia repair techniques in complex abdominal wall reconstruction. Am Surg 81:778-785

35. Lopez-Cano M, Armengol M, Quiles MT et al (2013) Preventive midline laparotomy closure with a new bioabsorbable mesh: an experimental study. J Surg Res 181:160-169

36. Veyrie N, Poghosyan T, Corigliano N, Canard G, Servajean S, Bouillot JL (2013) Lateral incisional hernia repair by the retromuscular approach with polyester standard mesh: topographic considerations and long-term followup of 61 consecutive patients. World J Surg 37:538-544. https://doi.org/10.1007/s00268-012-1857-9 